\title{
In-situ TEM Cryoindentation of Nanocrystalline Copper
}

\author{
Eric Lang ${ }^{1}$, Mike Marshall ${ }^{2}$, Henry Padilla ${ }^{2}$, Brad Boyce ${ }^{2}$ and Khalid Hattar ${ }^{2}$
}

${ }^{1}$ Sandia National Laboratories, Albuquerque, New Mexico, United States, ${ }^{2}$ Sandia National Laboratories, United States

In-situ TEM testing is a versatile platform for exploring nanoscale response to stimuli ranging from thermal, irradiation, corrosion, and stress environments. Advances in technologies have allowed for the development of TEM stages for integrated heating and cooling, straining, and liquid flow [1-5], allowing for the evolution of nanostructures to be observed in real time. In-situ TEM straining can be performed with various geometries and stress conditions. Prior work has been performed on tensile bar specimens, fracture bar bending specimens, nanopillar specimens, and wedge specimens. These different sample geometries allow for different mechanical properties to be extracted as the load-displacement data simultaneously collected with real-time video can be used to estimate to stresses and strains.

The mechanisms and fundamentals of nanoscale phenomena exhibited by nanocrystalline materials exhibiting nanoscale phenomena which influence bulk properties can be explored using in-situ TEM techniques. Cryogenic deformation of metals is a promising way to produce nanocrystalline materials [6]. Copper $(\mathrm{Cu})$ is an ideal material to study fundamental mechanical properties at low temperatures as it can exhibit microstructural changes such as grain growth at room temperature $[7,8]$. Cryogenic rolling of $\mathrm{Cu}$ is a technique for preparing fine-grained sheets with high strength and ductility by introducing nanoscale twins, shear bands, and kinks [8-10]. Thin films of $\mathrm{Cu}$ are of interest for tribology studies, as the microstructural changes under nanoscale plastic deformation can be used to determine the wear and friction properties $[10,11]$. However, there is a fundamental need to understand microstructural changes of $\mathrm{Cu}$ under tribological cryogenic loading conditions to correlate back with macroscopic mechanical properties to tailor microstructural features with desired applications in the future. Prior work has shown grain growth of nanocrystalline $\mathrm{Cu}$ following indentation at cryogenic temperatures [12]; however, a question remains: whether the growth occurs during the indentation or upon warming. This must be elucidated.

In this work, the cryoindentation response of $\mathrm{Cu}$ films is investigated with Gatan in-situ Cryo-Straining TEM holder (model \#671) run in reverse (Figure 1A). A Cu thin film was deposited by a Pulsed Laser Deposition (PLD) system onto silicon wedges. To create the desired strain effect via indentation, a unique dual wedge structure aligned perpendicular to each other ensured successful alignment of the indenter tip and the sample (Figure 1B). The wedges were fabricated from a silicon wafer by the tetramethyl ammonium hydroxide (TMAH) etching process to make a 55-degree wedge. When compressed together, the two sharp edges orient at right angles to each other contact generating high local strain at cryogenic temperatures. Both the sample and tip were held at about $-100{ }^{\circ} \mathrm{C}$ for one hour to equilibrate temperatures and ensure the indents were performed at this temperature. After indentation, the liquid nitrogen dewar was then emptied and the vacuum insulating the dewar was breached, allowing the stage to gradually warm within the microscope vacuum chamber.

The grain structures of $\mathrm{Cu}$ following indentation and warming to room temperature have been observed and analyzed. Dislocation pile-up is observed following the nanoindentation of the $\mathrm{Cu}$ film. Following warming to room temperature, slight grain growth is observed. Simultaneous videos recorded during the nanoindentation shows minimal microstructural changes as the warming progresses. These results indicate 
that grain growth occurs during the cryoindentation and not upon warming, mirroring the results seen by others with SEM-EBSD [13]. With the demonstration on the model material system of $\mathrm{Cu}$, we demonstrate that these techniques can be combined and show promise for use on more complex systems using recentlydeveloped in-situ TEM stages.

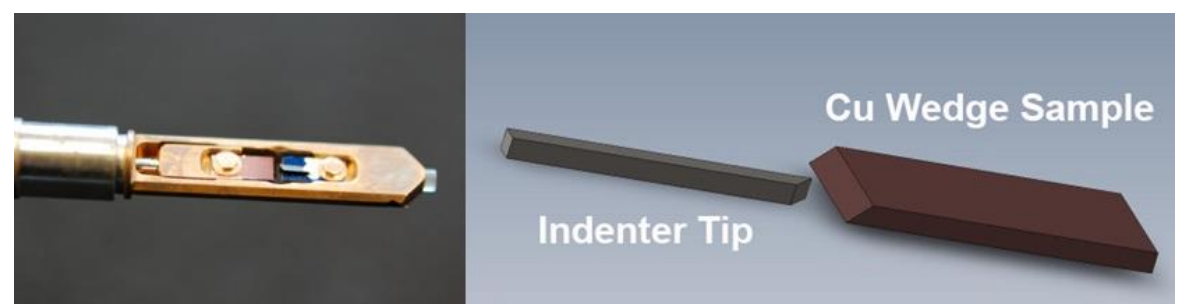

Figure 1. Fig. 1 (Left) The Gatan cryo straining tip. (Right) A unique dual wedge structure aligned perpendicular to each other ensured successful alignment of the indenter tip and the sample.

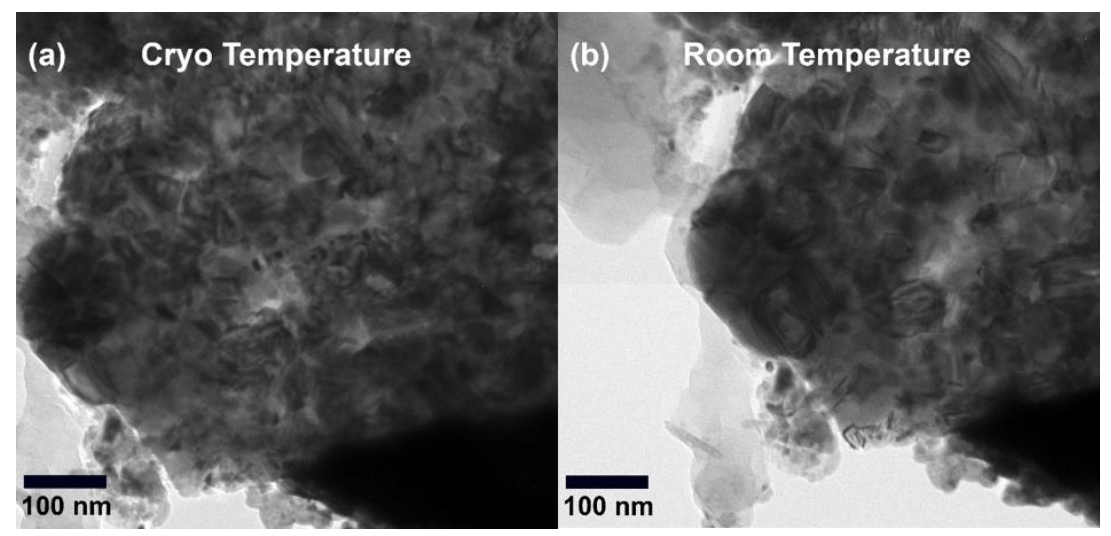

Figure 2. Fig. 2 TEM micrographs of the indented area of interest taken with sample at cryogenic temperatures (a) and after warming (b) showing changes to grain structure.

\section{References}

[1] K. Hattar, et al. Acta Materialia, 56 (2008), 794-801.

[2] K.L. Kurin, et al. J. Microscopy, 242 (2011), 117-123.

[3] J. Liu, et al. Material Characterization, 136 (2018), 331-336.

[4] Z. Fan, et al. Advanced Materials, 31 (2019), 1900608.

[5] A. Gouldstone, et al. Acta Materialia, 55 (2007), 4015-4039.

[6] Y. Huang, et al. Acta Materialia, 56 (2008), 1619.

[7] T. Konkova, et al. Scripta Materialia, 63 (2010), 921-924.

[8] W. Luo, et al. Material Letters, 157 (2015), 315-317.

[9] Y. Wang, et al. Nature, 419 (2002), 912-915.

[10] X.B. Li, et al. Material Science \& Engineering A, 772 (2020), 138811.

[11] X. Chen, et al. Acta Materialia, 161 (2018), 138-149.

[12] J.G. Brons, et al. Scripta Materialia 68 (2013) 781-784.

[13] D. Frazer, et al. Journal of Materials, 72 (2020), 2051-2056.

This work was supported by the US Department of Energy, Office of Basic Energy Sciences. Sandia National Laboratories is a multi-mission laboratory managed and operated by National Technology and Engineering Solutions of Sandia, LLC., a wholly owned subsidiary of Honeywell International, Inc., for the U.S. Department of Energy's National Nuclear Security Administration under contract DE-NA0003525 . 